\title{
Estudo normativo do sistema compreensivo do Rorschach para a cidade de São Paulo
}

\author{
Regina Sonia Gattas F. do Nascimento ${ }^{2}$
}

\begin{abstract}
Resumo
O método de Rorschach tem se mostrado bastante eficiente como instrumento na área de investigação e diagnóstico da personalidade. O sistema compreensivo de Exner tem garantido ao Rorschach status de instrumento de grande confiabilidade, por sua objetividade e precisão nos resultados. Entretanto, para que isso ocorra, é necessário trabalhar com normas apropriadas para a população. Por isso, realizamos uma pesquisa de normatização a fim de encontrar resultados para melhor utilização do sistema compreensivo em nosso meio. Aplicamos o Rorschach individualmente, em 200 sujeitos não-pacientes, de ambos os sexos, residentes na cidade de São Paulo, com idade variando entre 17 e 65 anos, de todos os graus de escolaridade e níveis socioeconômicos. Os resultados apresentam várias diferenças, quando comparados às normas apresentadas por Exner, mas algumas semelhanças com os estudos apresentados por especialistas de outros países, especialmente, os de origem latina. Estes resultados indicam influências culturais em diversas variáveis do Rorschach.

Palavras-Chave: Método de Rorschach; Sistema compreensivo; Estudo normativo; Cidade de São Paulo; Brasil.
\end{abstract}

\section{A normative research on the Rorschach comprehensive system in the city of São Paulo}

\begin{abstract}
The Rorschach method has proven quite efficient as an instrument for personality investigation and diagnosis. Comprehensive system has guaranteed the Rorschach the status of an instrument of great reliability because of its objectivity and the precision of its results. However, for this to occur, it is necessary to work with appropriate norms for a population. So, we carried out a normalization study in order to find results for the better utilization of the comprehensive system in our milieu. The Rorschach was applied individually in 200 non-patient subjects of both sexes, residents of the City of São Paulo, ages ranging from 17 to 65, of all levels of education and socioeconomic classes. The results show differences when compared to Exner's norms, but similarities to studies presented by specialists in other countries, especially those of a Latin origin. These results indicate the presence of cultural influences in Rorschach variables.
\end{abstract}

Keywords: Rorschach test; Comprehensive system; Normative study; City of São Paulo; Brazil.

\section{Introdução}

O Rorschach é um método de investigação de personalidade amplamente aceito entre os profissionais da psicologia e um dos mais valorizados por profissionais de outras áreas. No entanto, muitas vezes ainda é alvo de críticas e controvérsias.

Uma das questões que têm se discutido atualmente é a respeito do Rorschach tratar-se de instrumento perceptivo ou associativo. Weiner (1998) lembra que a monografia original de H. Rorschach, tinha como subtítulo "um teste de diagnóstico baseado na percepção". Weiner (1998) retoma também as propostas dos outros autores americanos que precederam o sistema compreensivo e destaca que Beck e Piotrowski priorizaram os aspectos perceptivos, lembrando que Beck "tomava as manchas como estímulos visuais $e$ as interpretações do Rorschach como um processo de quantificação das características da percepção" (p. 12). A respeito de Klopfer ressalta que, embora adepto da psicanálise, desenvolveu um sistema de avaliação das categorias formais das respostas do Rorschach, e desaconselhava a confiança excessiva nos conteúdos.

\footnotetext{
${ }^{1}$ Esta pesquisa teve o apoio financeiro da FAPESP. na área de informática de Rodney Franco de Lima.

Endereço para correspondência:

PUC: Faculdade de Psicologia - Rua Monte Alegre, 984 - São Paulo-SP - 01060-970

E-mail: rrecnascimento@,uol.com.br
}

${ }^{2}$ Colaboraram nesta pesquisa: Alexandre P. de Mattos, Armando Mori Neto, Carla C. Hisatugo, Flávia C. Mucarbel, Patrícia E. S. Calvaro; tivemos orientação para realizar os cálculos estatísticos da Profa. Yara Gustavo de Castro, da PUC-SP e o suporte 
A evolução contudo é notável. Houve um grande avanço na qualidade e aumento do poder estatístico das pesquisas com o método de Rorschach, como podemos observar no livro de Exner (1995) sobre metodologia de pesquisa com o Rorschach, bem como inúmeros trabalhos que aprimoram a compreensão dos aspectos mais subjetivos da avaliação das respostas. Entre estes, podemos citar o próprio sistema compreensivo, com suas novas variáveis para avaliação qualitativa dos conteúdos humanos (Exner, 2001), bem como diversos trabalhos com fundamentação psicanalítica para se trabalhar com os conteúdos das respostas, como apresentados por Lerner (1998). Esses novos modelos para avaliar aspectos qualitativos das respostas têm contribuído para uma análise mais objetiva dos conteúdos.

Weiner (1998) e Exner (1994), como outros rorschachistas contemporâneos, propõem a avaliação das categorias formais (elementos perceptivos), e das temáticas (elementos projetivos) de maneira integrada. Apesar desta discussão ter sido relativamente superada, ainda encontramos na literatura questionamentos a respeito da validade psicométrica do Rorschach, enquanto teóricos de linhas contrárias criticam o excesso de tratamento estatístico dos dados, e afirmam que o Rorschach não pode ser objeto desse tipo de análise, por considerar o método de maneira estritamente idiográfica.

Pensamos que esta afirmação de Exner (1994) sobre o assunto fecha a questão: "um examinador com experiência não incorrerá em nenbuma destas parcialidades, pois que ambas pressupõem um manejo mediocre do teste" (p. 260). Como o método inclui tanto elementos perceptivos quanto projetivos, conseguindo com estes avaliar os elementos das personalidades individuais (idiográficos) e, com os perceptivos, averiguar quanto as personalidades se parecem entre si (nomotéticos).

Uma outra polêmica relativa à compreensão dos resultados dos métodos de estudo da personalidade refere-se à consideração da universalidade dos seus resultados e à interferência de fatores culturais. Estudos recentes na área denominada psicologia cultural assinalam essa relação e afirmam que "processos mentais superiores, on o self, são inevitavelmente constituidos pela interaşão do indivíduo e cultura" (Piker, 1998, p. 20), o que também acontece com a expressão de emoções. A interação é de ambos os lados, uma vez que uma determinada cultura funciona e persiste mediante a organização das personalidades individuais.

Como afirmam Markus e Kitayama (1998),
... a perspectiva cultural assume que os processos psicológicos (...) não são apenas influenciados pela cultura, mas profundamente constituidos culturalmente. Por outro lado, a perspectiva cultural assume que as personalidades comportando-se em conjunto, criam a cultura. Cultura e personalidade são mais produtivamente analisados em conjunto, como apresentando uma dinâmica de mútua constituição. (p. 66)

Podemos concluir a esse respeito que tanto nas experiências da vida, como ao responder ao método de Rorschach, existe uma motivação derivada da pulsão (aspectos individuais) e ao mesmo tempo uma orientação e um controle para que a expressão seja legítima e consonante com os padrões de uma determinada cultura. A relação entre esses dois pólos é bastante complexa. E os especialistas devem estar preparados para compreender tanto a personalidade individual, quanto os elementos socioculturais que interferem nos padrões de suas respostas, pois o Rorschach, como todas as experiências humanas, revela ao mesmo tempo aspectos estritamente individuais e características que são próprias de seu grupo social e de sua cultura.

Nossa preocupação, neste estudo, tem como base aspectos relativos a determinantes culturais interferindo em resultados estruturais e objetivos do método de Rorschach. Lembramos que Weiner (1998) afirmou que para uma boa interpretação do Rorschach devemos ter uma aplicação correta, uma codificação bem conduzida (que a objetividade do sistema compreensivo possibilita) e normas adequadas para a população em questão.

Este trabalho teve como objetivo fazer uma análise descritiva de todas as variáveis do sistema compreensivo de Exner (média, desvio padrão, valores mínimo e máximo, mediana e moda de cada variável) de 200 indivíduos adultos não-pacientes, da cidade de São Paulo. A partir dos resultados, foram também levantadas as freqüências dos índices SCZI, DEPI, CDI, S-CON, OBS e HVI e a distribuição dos sujeitos nos diferentes tipos de vivência (EB). Como uma das etapas, para se chegar a um resultado mais confiável, foi também realizado um estudo de concordância entre codificadores, cujos resultados também serão relatados neste artigo.

Em razão do grande número de variáveis estudadas e por tratar-se de uma proposta de um trabalho breve, no final foram selecionadas apenas algumas variáveis para a discussão. O critério para essa seleção foi dar destaque a resultados discrepantes do estudo normativo apresentado por Exner (1999). 


\section{Método}

Sujeitos

Este trabalho consta de protocolos de 200 sujeitos adultos, residentes na cidade de São Paulo, com idade dos 17 até os 65 anos, de ambos os sexos, de todos os estados civis e graus de escolaridade, de diferentes níveis socioeconômicos, classificados pelo critério da ABIPEME, que não apresentem nenhuma queixa e/ou não estejam em tratamento psicológico ou psiquiátrico (a distribuição dos sujeitos encontra-se no Anexo 1). Esses indivíduos, com maioria de cor branca (80\%), possuem as mais variadas profissões e ocupações, entre elas, estudantes, pedreiros, bombeiros, secretárias, seminaristas, publicitários, do lar, médicos, músicos, advogados, dentistas, trabalhos de enfermagem, diversas atividades administrativas, etc.

\section{Procedimento}

Como é muito difícil trabalhar na cidade de São Paulo com uma amostra aleatória, procuramos ser bastante criteriosos na escolha dos sujeitos, inclusive com procedimento semelhante ao de Exner (1994, p. 199). Tentamos aproximar, da melhor forma possível, de uma amostra representativa.

Os participantes da pesquisa foram procurados em seus ambientes de trabalho, que por sua vez eram variados (banco, empresa de saúde, instituição pública, instituição de ensino, etc.) ou ambientes de estudo também variados (público, privado e de diferentes áreas). A idéia inicial era fazer um sorteio dentro das instituições, para compor os sujeitos da amostra. Contudo, este critério não se mostrou eficiente em determinadas empresas. Dessa forma, a amostra foi composta com maioria de sujeitos voluntários. Como Exner (1994), a equipe que realizou as aplicações do Rorschach procurou também alguns sujeitos voluntários, de ambientes variados, fora do ambiente de estudo ou de trabalho.

Foram feitas pequenas mudanças no procedimento, que se restringiram a instruções um pouco mais detalhadas, o que é importante para brasileiros que, diferentemente dos sujeitos americanos, possuem pouco contato com o método de Rorschach, e a sentarse em posição com ângulo de $90^{\circ}$ e não lateralmente, por ser mais habitual em nosso meio. No final da aplicação os sujeitos assinaram uma permissão para uso do material, de acordo com as atuais normas éticas para pesquisa com seres humanos.

Os profissionais que fizeram as aplicações dos protocolos de Rorschach eram psicólogos, com curso de especialização em Rorschach, seguindo o sistema compreensivo. As aplicações foram igualmente distribuídas entre os cinco psicólogos colaboradores desta pesquisa, que foram treinados pela coordenadora em reuniões com todo o grupo. Essa condição (vários aplicadores) garante maior validade à pesquisa, por evitar viés individual nesta fase do trabalho.

\section{Codificação e concordância entre codificadores}

A codificação de cada protocolo foi realizada por 2 pessoas separadamente, em combinações variadas. No caso de discordância, quase sempre a equipe de 5 pessoas, sob a orientação da coordenadora da pesquisa, decidiu pela melhor codificação.

Sabemos que os resultados de uma pesquisa com métodos projetivos podem refletir julgamentos idiossincráticos e subjetivos. No entanto, deve-se cuidar para realizar uma pesquisa confiável, ou seja, que apresente resultados objetivos que permitam sua reaplicação ou a generalização de seus resultados.

Para garantir maior confiabilidade para os resultados desta pesquisa, procuramos, em primeiro lugar, garantir julgamentos bastante consensuais entre a equipe de trabalho. Isto foi garantido pelas inúmeras reuniões para discussão das discordâncias entre dois codificadores, pela busca de regras apresentadas nos livros de Exner, nos exercícios por ele apresentados e pelo estabelecimento de algumas regras para determinadas codificações que se repetiram em nossa amostra e para as quais não encontramos referências na bibliografia.

Além desses cuidados, também foi realizado um estudo de concordância entre codificadores, pois de acordo com McDowell \& Acklin (1996, p. 310) "não há medida psicológica que não apresente erro". Este estudo, de acordo com os referidos autores, é fundamental para garantir validade, utilidade e poder das pesquisas com métodos projetivos.

Para realizar estes cálculos, foi efetuado um estudo por porcentagem simples de concordância e também com o coeficiente Kappa de concordância entre dois diferentes avaliadores (SPSS versão 8.0 para Windows). Para esta finalidade, foram selecionados $20 \%$ dos protocolos aplicados (40 protocolos no total), escolhidos ao acaso (a cada 5 protocolos tiramos um, sendo 8 de cada aplicador). Esses protocolos continham um total de 764 respostas, sendo cada uma delas considerada uma unidade de trabalho, ou segmento de análise. Foram codificados pela equipe da pesquisa, e por um juiz externo, considerado suficientemente preparado para uma codificação no sistema compreensivo. Foram escolhidos 3 diferentes juízes. As respostas foram divididas em todas as subcategorias de 
codificação, ou seja, localização, qualidade evolutiva, determinantes, qualidade formal, conteúdos, respostas populares, índice $\mathrm{Z}$ e códigos especiais. Foi realizada a análise de cada subcategoria separadamente.

\section{Resultados}

Os resultados do índice Kappa, relativos às concordâncias entre diferentes codificadores mostraramse bastante adequados, com muitos resultados acima de 0.80 . Destacam-se como valores abaixo de 0.40 os obtidos com as seguintes variáveis: $\mathrm{DQv} /+, \mathrm{Hx},(\mathrm{Ad})$, $\mathrm{Na}, \mathrm{DV}$, DR e FABCOM que foram categorias muito pouco freqüentes, mas ainda mostram uma tendência à concordância, com o nível de significância 0.000. Como absolutamente não confiáveis encontramos os índices $\mathrm{FQ}+$ e CONTAM. Os cálculos baseados na porcentagem simples de concordância apenas indicaram resultados abaixo de $80 \%$ em FQu $(76,4 \%)$ e dos valores atribuídos ao $\mathrm{Z}(77,9 \%)$.

Os resultados descritivos relativos às médias, desvios padrão, valores mínimos e máximos, modas e medianas de cada variável encontram-se na Tabela 5 (Anexo 2). Esses cálculos foram realizados por meio do programa de estatística do RIAP 4 plus, executado diretamente a partir do banco de dados, o que garante maior confiabilidade, pois evita a construção de tabelas. As freqüências dos índices SCZI, DEPI, CDI, S-CON, OBS E HVI considerados positivos na amostra encontram-se no Anexo 3.

\section{Discussão}

Primeiramente gostaríamos de salientar a grande discrepância dos resultados em relação aos padrões americanos. Estes resultados, contudo, não são tão diferentes quando observamos outros resultados normativos ("Tables for the International Symposium on the Rorschach Nonpatient Data: findings from around the world I, II, III"XVI Congresso Internacional de Rorschach - 1999).

Como já foi dito anteriormente, comentaremos apenas alguns desses resultados. Os comentários referem-se às discrepâncias em relação às normas de Exner (1999).

Em primeiro lugar, chama a atenção o número de respostas (R). Embora a média [São Paulo $(S P)=20,40$, amer. $=23]$ seja relativamente próxima dos resultados americanos, a nossa mediana é bem mais baixa $(\mathrm{SP}=18,00$, amer. $=22,67)$, e a moda é bastante peculiar $(\mathrm{SP}=14$, amer. $=23)$. Este resultado acarretou diferenças em praticamente todo o restante, uma vez que com número tão baixo de respostas todas as freqüências ficam alteradas. Foi realizado então um estudo estatístico da diferença entre as médias, separando o grupo pela mediana do $\mathrm{R}$, onde foram encontradas muitas diferenças significativas, o que era de se esperar. Em virtude dessas discrepâncias, consideramos mais adequado (o que foi aprovado por John Exner pessoalmente) trabalhar com duas tabelas diferentes, conforme Tabelas 12 e 13 (Anexos 4 e 5), considerando $\mathrm{R}$ uma variável "confundidora" em nosso meio. Acreditamos que a falta de experiência dos sujeitos com avaliação psicológica e o pouco conhecimento que se tem do Rorschach em nosso meio possam ter contribuído para esse resultado. Além desse fator, devemos lembrar que nossa amostra inclui sujeitos com baixo nível de escolaridade, o que não acontece com a amostra americana. É interessante que apesar de tantas discrepâncias entre os dois grupos, não são significativas as diferenças de Lambda, AdjD e X-\%, o que nos dá a idéia que não se refere a algum desajustamento, mas apenas um estilo de responder. A distribuição de GHR e PHR em ambos os grupos (protocolos longos: GHR: média $=3.07$; $\operatorname{moda}=2,0 \mathrm{e}$ PHR: média $=3,73$; moda $=2,0$, enquanto que nos protocolos curtos GHR: média $=2,39 ;$ moda $=2,0$ e PHR: média $=1,80 ;$ moda $=0,50)$ confirmam esta mesma tendência. Pretendemos nos aprofundar futuramente na compreensão desta distribuição de respostas.

Também se salientam os valores encontrados em EA, es, D e AdjD. EA encontra-se mais baixo do que na população americana $(\mathrm{SP}=4,96$; amer. $=8,82)$, enquanto es apresenta uma média muito semelhante $(\mathrm{SP}=8,57$; amer.=8,21). Os valores de $\mathrm{D}$ e AdjD são negativos, indicando a presença de uma situação estressante, que é mais acentuada como variável situacional. Talvez a própria situação de prova possa ser mais estressante do que em outros grupos culturais.

Acreditamos no entanto que esses resultados indicam que na cidade de São Paulo estamos vivendo um clima de estresse e insegurança que provavelmente ultrapassa os níveis suportáveis individualmente e não há compensações para essa situação, ou seja, para boa parte das pessoas não há uma estrutura familiar e relacional que possa ser compensatória para garantir o equilíbrio emocional. As pessoas acabam por se afastar dos relacionamentos - como foi identificado em outros trabalhos com parte desta amostra (Mucarbel e Nascimento, 2001a e 2001b) - e demonstram grande vulnerabilidade para enfrentar as tensões próprias da vida cotidiana. Para dar continuidade a este estudo, pretendemos estudar a distribuição dos resultados em 
outras cidades do estado de São Paulo. Embora não manifestem freqüentemente a tendência a isolar-se (Isolate - média $\mathrm{SP}=0,24$, amer. $=0,20 ;$ moda $\mathrm{SP}=0$, amer. $=0,29)$, os resultados das respostas de textura $(T)$ são baixos. A média de T é $<1$ e a moda e mediana são iguais a zero. São portanto pessoas que tendem a manter um certo afastamento em suas relações interpessoais.

Quanto aos resultados da Qualidade Formal, já tivemos oportunidade de os apresentar (Nascimento e colab., 2000). Neste trabalho queremos apenas salientar o fato de haver constatado posteriormente à conclusão desta pesquisa, em contatos com especialistas internacionais do sistema compreensivo, que nossa avaliação tem sido mais rigorosa do que a dos americanos, especialmente nas situações em que há múltiplos objetos, em que nem todos têm a mesma qualidade formal. A regra para estes casos é atribuir a qualidade menos favorável (Exner, 1999, p. 56). O uso deste critério faz com que fiquem aumentados os valores de $\mathrm{Xu} \%+$ e X-\%. No entanto, sendo pouco freqüente a utilização desta regra, não acreditamos que os resultados apresentaram tanta diferença em relação a médias fornecidas por Exner (1999), apenas em virtude do uso deste critério. A distribuição dos introversivos (31,5\%), extratensivos (19,5\%) e ambiguais (49\%), também é muito diferente daquela da população americana, com uma proporção muito alta de ambiguais. Este resultado é ainda mais acentuado quando consideramos os casos com $\mathrm{R}<19$. Isso se deve ao fato do EA sempre muito baixo elevar os casos de ambiguais. Chama a atenção também o fato de serem mais freqüentes pessoas introversivas do que extratensivas, o que não faz parte do estereótipo dos brasileiros.

Quanto aos índices, há três muito freqüentes: DEPI, CDI, e SCZI. O DEPI (24\% da amostra), já foi estudado separadamente e foi apresentado no congresso da ALAR (Nascimento e Güntert, 2001).

O CDI (positivo em 54,5\% da amostra), merece um estudo especial, mas por ora, podemos pensar que esteja associado ao baixo número de respostas de grande parte do grupo. No entanto, também nos indica tratar-se de um grupo com pouca habilidade para relacionamentos interpessoais, o que mais uma vez destoa do estereótipo do brasileiro, que é considerado um povo de boas relações com as pessoas, o que não foi confirmado em vários resultados deste estudo.

Também merece atenção o $S C Z I$, presente em $21 \%$ da amostra. Não pretendemos mais estudar detalhadamente este índice, uma vez que já sofreu alterações no sistema compreensivo. O que podemos afirmar após ter avaliado os protocolos que obtivemos é que não encontramos tantas pessoas com características psicóticas. Podem sim, em alguns casos, tratar-se de falsos positivos e algumas poucas vezes de positivos. No entanto, por causa das diferenças de normas para as duas populações, fica claro que este índice não é adequado para o nosso grupo.

$\mathrm{Na}$ situação oposta encontram-se os obsessivos, uma vez que não foi encontrado nenhum caso de pessoa com este estilo (OBS) e atribuímos ao fato de termos uma quantidade rebaixada de respostas FQXo e aumentada a de FQXu. Dessa forma, muito poucas vezes pudemos atribuir FQX+ e nossa média de $\mathrm{X}+\%$ também é baixa, o que acarreta em não se poder confiar também nos critérios deste índice para população que não apresente a mesma distribuição das respostas do grupo de Exner.

Ainda não estudamos a distribuição do PTI, mas já se pode notar uma distribuição mais razoável dos índices $\mathrm{WDA} \%(0,78 \%)$ e $\mathrm{XA} \% \quad(0,74 \%)$, se comparados ao $\mathrm{X}+\%$. Estas médias, embora diferentes das respectivas americanas $(0,94$ e 0,92$)$, ficam fora dos limites indicadores de distúrbios de percepção e pensamento, o que facilita o uso do PTI.

\section{Conclusão}

A prática clínica já vinha fornecendo parâmetros relativos às diferenças em relação ao grupo americano. No entanto, esta pesquisa com sujeitos não pacientes possibilitou maior esclarecimento referente aos resultados do Rorschach, no sistema compreensivo, em nosso meio.

Considerando o conjunto dos resultados obtidos até o momento, fica evidente que as tabelas de Exner (1999) não são totalmente adequadas para avaliação de nossos protocolos. Embora ainda não esteja concluída esta investigação, acreditamos já termos resultados mais adequados para nossa população. Mais especificamente, adequados para a população da cidade de São Paulo. Esperamos também que outros projetos sejam realizados em outras regiões do Brasil, para integrar com estes resultados e termos resultados que representem melhor nosso país.

Sabemos que um especialista competente saberá em qualquer situação conduzir o seu trabalho na ausência de pesquisas adequadas. Contudo, para que o Rorschach seja utilizado de forma ampla, completa e segura, deve basear-se em normas adequadas.

Podemos ainda compreender estes resultados a partir das novas propostas dos estudiosos da psicologia intercultural. Por este ângulo, é possível afirmar que 
embora o Rorschach seja capaz de identificar características universais de personalidade, sempre será necessário realizar pesquisas para se verificarem diferenças, estudando, por exemplo, os diferentes níveis socioeconômicos, ou os diferentes graus de instrução, o que pretendemos concretizar brevemente.

A leitura destas tabelas constitui-se agora um desafio. Quando considerar patológica a presença de um índice que indica uma dificuldade de adaptação, ou até mesmo uma patologia, quando este índice foi encontrado com bastante freqüência em uma população de não-pacientes?

\section{Referência}

Congresso Internacional de Rorschach (XVI) (1999). "Tables for the International Symposium on the Rorschach Nonpatient Data: findings from around the world I, II, III". Amsterdã.

Exner, J. E. (1994). El Rorschach, un Sistema Comprehensivo, vol. I. Rorschach Workshops. Madrid: Psimática.

Exner, J. E. (1995). Issues and Methods in Rorschach Research. New Jersey: Lawrence Erlbaum Associates Publishers.

Exner, J. E. (1999). Manual de Classificação do Rorschach para o sistema compreensivo. São Paulo: Casa do Psicólogo.

Exner, J. E. (2001). A Rorschach Workbook for the Comprehensive System. Fifth Edition. Asheville: Rorschach Workshops.

Lerner, P. M. (1998). Psychoanalytic Perspectives on the Rorschach. Hillsdale: The Analytic Press.

Markus, H. R. \& Kitayama, S. (1998). The Cultural Psychology of Personality. Journal of Cross-Cultural Psychology, v. 29, n. 1, 63-87.
McDowell, C. J. \& Acklin, M. W. (1996). Standardizing Procedures for Calculating Rorschach Interrater Reliability: Conceptual and Empirical Foundations. Journal of Personality Assessment, 66(2), 308-320.

Mucarbel, F. C. \& Nascimento, R. S. G. F. (2001a). Análise das Relações Interpessoais por meio do Método de Rorschach: um estudo das respostas da prancha III. Libro de Resúmenes del XI Congreso Latinoamericano de Rorschach y otras técnicas proyectivas da ALAR, Rosário, Argentina, p. 113.

Mucarbel, F. C. \& Nascimento, R. S. G. F. (2001b). Avaliação da Variável de Representação Humana do método de Rorschach, segundo o Sistema Compreensivo: um estudo das respostas GHR e PHR. IV Encontro da Sociedade Brasileira de Rorschach e outras técnicas de Avaliação Psicológica - SBRo. Itatiba, SP.

Nascimento, R. S. G. F. e colaboradores (2000). Um Estudo da Qualidade Formal em um Grupo de Sujeitos Não-Pacientes de São Paulo, conforme o Sistema Compreensivo de Exner. Em C. Vaz, - Livro dos Anais. Porto Alegre: AGE, p. 40-49.

Nascimento, R. S. G. \& Güntert, A. E. V. A. (2001). O índice DEPI do sistema compreensivo na população brasileira. Libro de Resúmenes del XI Congresso Latinoamericano de Rorschach y otras Técnicas Proyectivas da ALAR, Rosário, Argentina, p. 129.

Piker, S. (1998). Contributions of Psychological Anthropology. Journal of Cross-Cultural Psychology, v. 29, n. 1, 9-31.

Weiner, I. B. (1998). Principles of Rorschach interpretation. Mahwah, New Jersey: Lawrence Erlbaum Associates Publishers. 


\section{Anexo 1}

Distribuição dos Sujeitos

\begin{tabular}{lcc}
\hline \multicolumn{3}{c}{ Tabela 1 } \\
\hline Idade & $\mathrm{f}$ & $\%$ \\
$17-26$ & 69 & 35 \\
$27-36$ & 74 & 37 \\
$37-46$ & 38 & 19 \\
Acima de 47 & 19 & 10 \\
\hline
\end{tabular}

\begin{tabular}{lcc}
\hline \multicolumn{3}{c}{ Tabela 2} \\
\hline Sexo & F & $\%$ \\
MASCULINO & 95 & 48 \\
FEMININO & 105 & 53 \\
& & \\
\hline
\end{tabular}

\begin{tabular}{lcc}
\hline & Tabela 3 & \\
\hline Educação & $\mathrm{f}$ & $\%$ \\
$<$ de 8 anos & 42 & 21 \\
$9-11$ anos & 62 & 31 \\
$12-15$ anos & 55 & 28 \\
$16+$ anos & 41 & 21 \\
\hline
\end{tabular}

\begin{tabular}{ccc}
\hline \multicolumn{3}{c}{ Tabela 4 } \\
\hline Nível Socioeconômico & $\mathrm{f}$ & $\%$ \\
A & 29 & 15 \\
B & 77 & 38 \\
C & 66 & 33 \\
D & 20 & 10 \\
E & 8 & 4 \\
\hline
\end{tabular}

\section{Anexo 2}

Tabela 5 - RIAP Estatística descritiva para 200 sujeitos

\begin{tabular}{lccccccc}
\multicolumn{1}{c}{ Continua } \\
\hline Variável & Média & DP & Mínimo & Máximo & Freqüência & Mediana & Moda \\
Idade & 31.06 & 9.89 & 17.00 & 59.00 & 200 & 30.00 & -- \\
Yrs. Ed. & 11.73 & 4.60 & 0.00 & 23.00 & 199 & 11.00 & 11.00 \\
R & 20.40 & 6.84 & 14.00 & 50.00 & 200 & 18.00 & 14.00 \\
W & 7.69 & 4.00 & 0.00 & 19.00 & 198 & 7.00 & 6.00 \\
D & 8.72 & 4.52 & 0.00 & 27.00 & 198 & 8.00 & 9.00 \\
Dd & 4.00 & 3.31 & 0.00 & 19.00 & 180 & 3.00 & 3.00 \\
S & 1.77 & 1.73 & 0.00 & 10.00 & 151 & 1.00 & 1.00 \\
DQ+ & 4.76 & 3.00 & 0.00 & 15.00 & 190 & 4.00 & 4.00 \\
DQo & 13.90 & 6.15 & 4.00 & 41.00 & 200 & 12.00 & 12.00 \\
DQv & 1.43 & 1.83 & 0.00 & 12.00 & 121 & 1.00 & 0.00 \\
DQv/+ & 0.32 & 0.74 & 0.00 & 6.00 & 46 & 0.00 & 0.00 \\
FQx+ & 0.19 & 0.46 & 0.00 & 2.00 & 31 & 0.00 & 0.00 \\
FQxo & 8.70 & 3.44 & 3.00 & 23.00 & 200 & 9.00 & 9.00 \\
FQxu & 6.15 & 3.45 & 1.00 & 22.00 & 200 & 5.00 & 5.00 \\
FQx- & 5.06 & 2.75 & 0.00 & 16.00 & 199 & 5.00 & 4.00 \\
FQx_none & 0.31 & 0.78 & 0.00 & 6.00 & 41 & 0.00 & 0.00 \\
MQual + & 0.08 & 0.27 & 0.00 & 1.00 & 16 & 0.00 & 0.00 \\
MQual o & 1.57 & 1.42 & 0.00 & 8.00 & 156 & 1.00 & 1.00 \\
MQual u & 0.59 & 0.88 & 0.00 & 5.00 & 83 & 0.00 & 0.00 \\
MQual - & 0.60 & 1.03 & 0.00 & 5.00 & 69 & 0.00 & 0.00
\end{tabular}

Psico-USF, v. 7, n. 2, p. 127-141, Jul./Deそ: 2002 
Tabela 5 - RIAP Estatística descritiva para 200 sujeitos

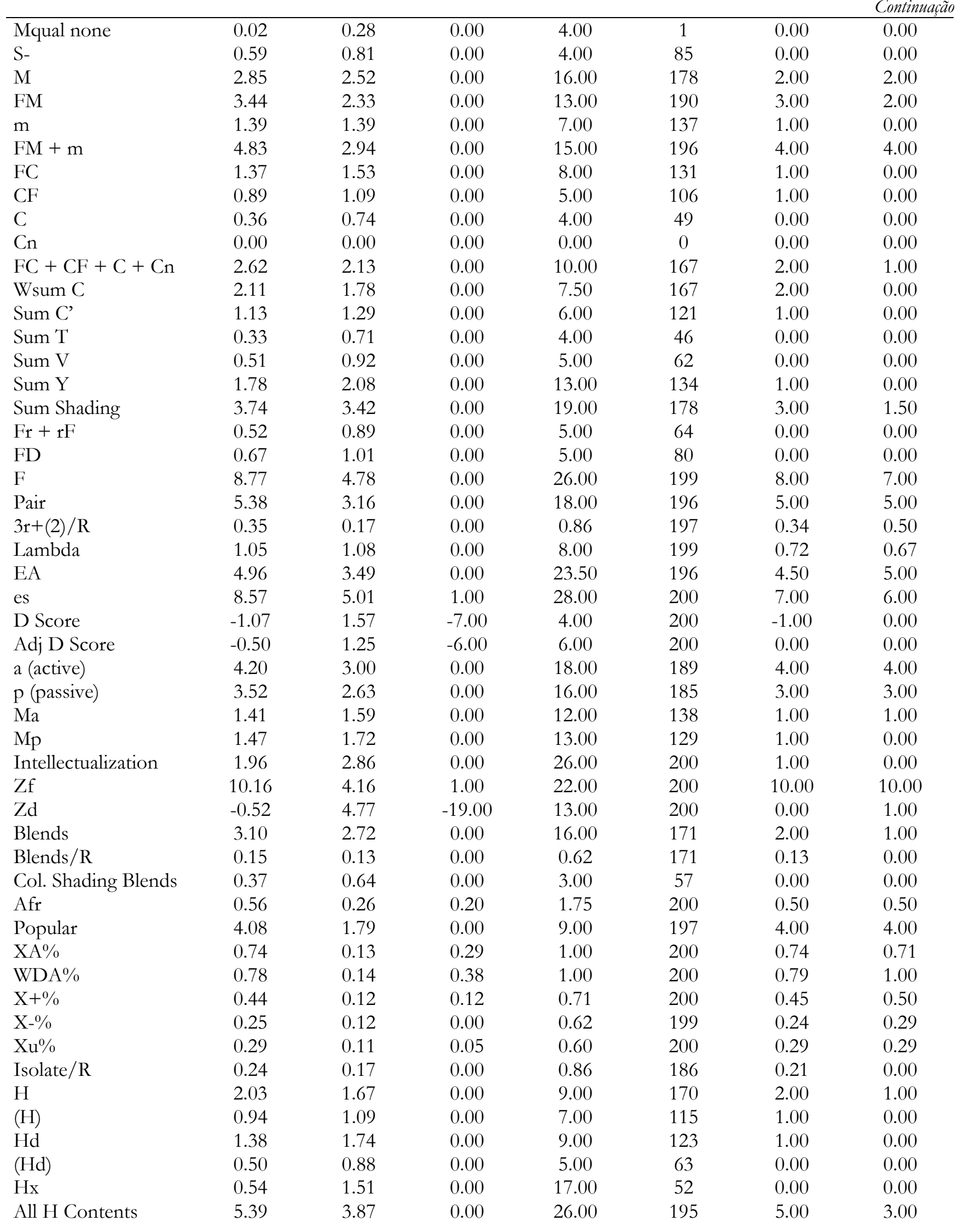


Tabela 5 - RIAP Estatística descritiva para 200 sujeitos

\begin{tabular}{|c|c|c|c|c|c|c|c|}
\hline & & & & & & & \\
\hline A & 7.76 & 2.91 & 3.00 & 17.00 & 200 & 7.00 & 6.00 \\
\hline (A) & 0.32 & 0.55 & 0.00 & 2.00 & 54 & 0.00 & 0.00 \\
\hline Ad & 1.61 & 1.57 & 0.00 & 9.00 & 146 & 1.00 & 1.00 \\
\hline (Ad) & 0.08 & 0.27 & 0.00 & 1.00 & 16 & 0.00 & 0.00 \\
\hline An & 1.24 & 1.64 & 0.00 & 10.00 & 114 & 1.00 & 0.00 \\
\hline Art & 0.97 & 1.21 & 0.00 & 6.00 & 103 & 1.00 & 0.00 \\
\hline Ay & 0.28 & 0.64 & 0.00 & 4.00 & 42 & 0.00 & 0.00 \\
\hline $\mathrm{Bl}$ & 0.22 & 0.67 & 0.00 & 6.00 & 29 & 0.00 & 0.00 \\
\hline $\mathrm{Bt}$ & 1.50 & 1.37 & 0.00 & 6.00 & 142 & 1.00 & 0.00 \\
\hline $\mathrm{Cg}$ & 1.29 & 1.43 & 0.00 & 7.00 & 126 & 1.00 & 0.00 \\
\hline $\mathrm{Cl}$ & 0.34 & 0.68 & 0.00 & 4.00 & 49 & 0.00 & 0.00 \\
\hline $\mathrm{Ex}$ & 0.20 & 0.50 & 0.00 & 3.00 & 32 & 0.00 & 0.00 \\
\hline $\mathrm{Fi}$ & 0.36 & 0.71 & 0.00 & 4.00 & 51 & 0.00 & 0.00 \\
\hline $\mathrm{Fd}$ & 0.26 & 0.54 & 0.00 & 3.00 & 43 & 0.00 & 0.00 \\
\hline $\mathrm{Ge}$ & 0.22 & 0.58 & 0.00 & 3.00 & 32 & 0.00 & 0.00 \\
\hline $\mathrm{Hh}$ & 0.42 & 0.75 & 0.00 & 4.00 & 60 & 0.00 & 0.00 \\
\hline Ls & 0.77 & 0.96 & 0.00 & 5.00 & 100 & 0.50 & 0.00 \\
\hline $\mathrm{Na}$ & 0.83 & 1.02 & 0.00 & 5.00 & 100 & 0.50 & 0.00 \\
\hline $\mathrm{Sc}$ & 1.01 & 1.32 & 0.00 & 8.00 & 109 & 1.00 & 0.00 \\
\hline Sx & 0.32 & 0.82 & 0.00 & 5.00 & 36 & 0.00 & 0.00 \\
\hline Xy & 0.20 & 0.50 & 0.00 & 3.00 & 32 & 0.00 & 0.00 \\
\hline Id & 0.79 & 1.05 & 0.00 & 5.00 & 94 & 0.00 & 0.00 \\
\hline $\mathrm{DV}$ & 1.05 & 1.34 & 0.00 & 7.00 & 107 & 1.00 & 0.00 \\
\hline INCOM & 0.64 & 0.90 & 0.00 & 4.00 & 84 & 0.00 & 0.00 \\
\hline DR & 0.78 & 1.25 & 0.00 & 7.00 & 85 & 0.00 & 0.00 \\
\hline FABCOM & 0.41 & 0.78 & 0.00 & 4.00 & 58 & 0.00 & 0.00 \\
\hline DV2 & 0.05 & 0.25 & 0.00 & 2.00 & 7 & 0.00 & 0.00 \\
\hline INCOM2 & 0.16 & 0.47 & 0.00 & 3.00 & 25 & 0.00 & 0.00 \\
\hline DR2 & 0.09 & 0.48 & 0.00 & 5.00 & 10 & 0.00 & 0.00 \\
\hline FABCOM2 & 0.04 & 0.20 & 0.00 & 1.00 & 8 & 0.00 & 0.00 \\
\hline ALOG & 0.50 & 0.84 & 0.00 & 4.00 & 66 & 0.00 & 0.00 \\
\hline CONTAM & 0.05 & 0.38 & 0.00 & 5.00 & 5 & 0.00 & 0.00 \\
\hline Sum6 Sp. Scores & 3.75 & 3.04 & 0.00 & 17.00 & 183 & 3.00 & --- \\
\hline Lvl-2 Sp. Scores & 0.34 & 0.87 & 0.00 & 7.00 & 42 & 0.00 & 0.00 \\
\hline WSum6 & 10.63 & 11.08 & 0.00 & 85.00 & 183 & 8.00 & 0.00 \\
\hline $\mathrm{AB}$ & 0.36 & 1.19 & 0.00 & 13.00 & 35 & 0.00 & 0.00 \\
\hline $\mathrm{AG}$ & 0.27 & 0.66 & 0.00 & 4.00 & 38 & 0.00 & 0.00 \\
\hline COP & 0.63 & 0.94 & 0.00 & 4.00 & 79 & 0.00 & 0.00 \\
\hline $\mathrm{CP}$ & 0.05 & 0.21 & 0.00 & 1.00 & 9 & 0.00 & 0.00 \\
\hline GHR & 2.72 & 1.92 & 0.00 & 9.00 & 179 & 2.00 & 2.00 \\
\hline PHR & 2.73 & 2.63 & 0.00 & 15.00 & 164 & 2.00 & 1.00 \\
\hline MOR & 0.77 & 1.24 & 0.00 & 7.00 & 84 & 0.00 & 0.00 \\
\hline PER & 0.47 & 0.88 & 0.00 & 5.00 & 60 & 0.00 & 0.00 \\
\hline PSV & 0.26 & 0.59 & 0.00 & 3.00 & 39 & 0.00 & 0.00 \\
\hline
\end{tabular}


Anexo 3

Freqüência dos Índices : Adultos Não-Pacientes de São Paulo (N=200)

Tabela 6 - SCZI

\begin{tabular}{c|cccc}
\hline Válidos & Freqüência & Porcentagem & Porcentagem Válida & Porcentagem Cumulativa \\
\hline 0 & 158 & 79,0 & 79,0 & 79,0 \\
1 & 42 & 21,0 & 21,0 & 100,0 \\
Total & 200 & 100,0 & 100,0 & \\
\hline
\end{tabular}

Tabela 7 - DEPI

\begin{tabular}{c|cccc}
\hline Válidos & Freqüência & Porcentagem & Porcentagem Válida & Porcentagem Cumulativa \\
\hline 0 & 152 & 76,0 & 76,0 & 76,0 \\
1 & 48 & 24,0 & 24,0 & 100,0 \\
\hline Total & 200 & 100,0 & 100,0 & \\
\hline
\end{tabular}

Tabela 8 -CDI

\begin{tabular}{c|cccc}
\hline Válidos & Freqüência & Porcentagem & Porcentagem Válida & Porcentagem Cumulativa \\
\hline 0 & 91 & 45,5 & 45,5 & 45,5 \\
1 & 109 & 54,5 & 54,5 & 100,0 \\
\hline Total & 200 & 100,0 & 100,0 & \\
\hline
\end{tabular}

Tabela 9 - S-CON

\begin{tabular}{c|cccc}
\hline Válidos & Freqüência & Porcentagem & Porcentagem Válida & Porcentagem Cumulativa \\
\hline 0 & 192 & 96,0 & 96,0 & 96,0 \\
1 & 8 & 4,0 & 4,0 & 100,0 \\
Total & 200 & 100,0 & 100,0 & \\
\hline
\end{tabular}

Tabela 10 - HVI

\begin{tabular}{c|cccc}
\hline Válidos & Freqüência & Porcentagem & Porcentagem Válida & Porcentagem Cumulativa \\
\hline 0 & 182 & 91,0 & 91,0 & 91,0 \\
1 & 18 & 9,0 & 9,0 & 100,0 \\
Total & 200 & 100,0 & 100,0 & \\
\hline
\end{tabular}

Tabela 11 - OBS

\begin{tabular}{c|cccc}
\hline Válidos & Freqüência & Porcentagem & Porcentagem Válida & Porcentagem Cumulativa \\
\hline 0 & 200 & 100,0 & 100,0 & 100,0 \\
\hline
\end{tabular}




\section{Anexo 4}

Tabela 12 - RIAP Estatística descritiva para 104 protocolos selecionados: $\mathrm{R}<19$

\begin{tabular}{|c|c|c|c|c|c|c|c|}
\hline Variável & Média & DP & Mínimo & Máximo & Freqüência & Mediana & Moda \\
\hline Idade & 30.71 & 10.24 & 17.00 & 59.00 & 104 & 28.50 & 30.00 \\
\hline Yrs. Ed. & 9.26 & 6.11 & 0.00 & 21.00 & 87 & 11.00 & 11.00 \\
\hline $\mathrm{R}$ & 15.73 & 1.44 & 14.00 & 18.00 & 104 & 16.00 & 14.00 \\
\hline W & 6.63 & 3.03 & 0.00 & 14.00 & 103 & 7.00 & 7.00 \\
\hline $\mathrm{D}$ & 6.23 & 2.65 & 0.00 & 13.00 & 102 & 6.00 & 6.00 \\
\hline Dd & 2.88 & 2.12 & 0.00 & 9.00 & 89 & 3.00 & 2.00 \\
\hline S & 1.41 & 1.24 & 0.00 & 5.00 & 75 & 1.00 & 1.00 \\
\hline DQ+ & 4.15 & 2.31 & 0.00 & 10.00 & 100 & 4.00 & 4.00 \\
\hline DQo & 10.41 & 2.62 & 4.00 & 18.00 & 104 & 11.00 & 12.00 \\
\hline DQv & 0.88 & 1.23 & 0.00 & 7.00 & 50 & 0.00 & 0.00 \\
\hline DQv/+ & 0.28 & 0.58 & 0.00 & 3.00 & 24 & 0.00 & 0.00 \\
\hline FQx+ & 0.17 & 0.43 & 0.00 & 2.00 & 16 & 0.00 & 0.00 \\
\hline FQxo & 7.04 & 2.11 & 3.00 & 11.00 & 104 & 7.50 & 9.00 \\
\hline FQxu & 4.51 & 1.75 & 1.00 & 9.00 & 104 & 4.00 & 3.50 \\
\hline FQx- & 3.87 & 2.11 & 0.00 & 10.00 & 103 & 4.00 & 3.00 \\
\hline FQx_none & 0.14 & 0.43 & 0.00 & 2.00 & 12 & 0.00 & 0.00 \\
\hline MQual + & 0.07 & 0.25 & 0.00 & 1.00 & 7 & 0.00 & 0.00 \\
\hline MQual o & 1.29 & 1.19 & 0.00 & 6.00 & 77 & 1.00 & 1.00 \\
\hline MQual u & 0.45 & 0.78 & 0.00 & 4.00 & 34 & 0.00 & 0.00 \\
\hline MQual - & 0.39 & 0.73 & 0.00 & 4.00 & 30 & 0.00 & 0.00 \\
\hline MQual none & 0.00 & 0.00 & 0.00 & 0.00 & 0 & 0.00 & 0.00 \\
\hline S- & 0.49 & 0.64 & 0.00 & 2.00 & 43 & 0.00 & 0.00 \\
\hline M & 2.20 & 1.64 & 0.00 & 6.00 & 90 & 2.00 & 2.00 \\
\hline FM & 3.10 & 1.96 & 0.00 & 12.00 & 101 & 3.00 & --- \\
\hline $\mathrm{m}$ & 1.16 & 1.20 & 0.00 & 5.00 & 67 & 1.00 & 0.00 \\
\hline $\mathrm{FM}+\mathrm{m}$ & 4.26 & 2.39 & 0.00 & 12.00 & 102 & 4.00 & 4.00 \\
\hline FC & 0.94 & 1.10 & 0.00 & 5.00 & 59 & 1.00 & 0.00 \\
\hline CF & 0.72 & 0.95 & 0.00 & 4.00 & 48 & 0.00 & 0.00 \\
\hline C & 0.25 & 0.55 & 0.00 & 2.00 & 20 & 0.00 & 0.00 \\
\hline $\mathrm{Cn}$ & 0.00 & 0.00 & 0.00 & 0.00 & 0 & 0.00 & 0.00 \\
\hline $\mathrm{FC}+\mathrm{CF}+\mathrm{C}+\mathrm{Cn}$ & 1.91 & 1.58 & 0.00 & 7.00 & 81 & 2.00 & 1.00 \\
\hline WSum C & 1.57 & 1.38 & 0.00 & 6.50 & 81 & 1.50 & 0.00 \\
\hline Sum C' & 0.84 & 1.17 & 0.00 & 5.00 & 49 & 0.00 & 0.00 \\
\hline Sum $\mathrm{T}$ & 0.20 & 0.49 & 0.00 & 3.00 & 18 & 0.00 & 0.00 \\
\hline Sum V & 0.33 & 0.75 & 0.00 & 3.00 & 21 & 0.00 & 0.00 \\
\hline Sum Y & 1.20 & 1.61 & 0.00 & 8.00 & 59 & 1.00 & 0.00 \\
\hline Sum Shading & 2.57 & 2.34 & 0.00 & 9.00 & 85 & 2.00 & 1.50 \\
\hline $\mathrm{Fr}+\mathrm{rF}$ & 0.47 & 0.71 & 0.00 & 3.00 & 37 & 0.00 & 0.00 \\
\hline FD & 0.73 & 0.98 & 0.00 & 4.00 & 47 & 0.00 & 0.00 \\
\hline F & 6.59 & 3.07 & 0.00 & 16.00 & 103 & 6.50 & 6.50 \\
\hline Pair & 4.30 & 2.24 & 0.00 & 11.00 & 100 & 4.00 & 4.00 \\
\hline $3 r+(2) / \mathrm{R}$ & 0.36 & 0.18 & 0.00 & 0.80 & 101 & 0.35 & 0.50 \\
\hline Lambda & 1.03 & 1.14 & 0.00 & 8.00 & 103 & 0.70 & --- \\
\hline EA & 3.77 & 2.33 & 0.00 & 11.50 & 101 & 3.50 & 1.00 \\
\hline es & 6.83 & 3.59 & 1.00 & 15.00 & 104 & 6.00 & 4.00 \\
\hline D Score & -0.87 & 1.26 & -5.00 & 2.00 & 104 & -1.00 & 0.00 \\
\hline Adj D Score & -0.51 & 1.03 & -4.00 & 2.00 & 104 & 0.00 & 0.00 \\
\hline
\end{tabular}


Tabela 12 - RIAP Estatística descritiva para 104 protocolos selecionados: R <19

\begin{tabular}{|c|c|c|c|c|c|c|c|}
\hline & & & & & & & Contin \\
\hline a (active) & 3.72 & 2.53 & 0.00 & 13.00 & 97 & 3.50 & 4.00 \\
\hline $\mathrm{p}$ (passive) & 2.76 & 1.83 & 0.00 & 8.00 & 95 & 3.00 & 3.00 \\
\hline $\mathrm{Ma}$ & 1.14 & 1.16 & 0.00 & 5.00 & 69 & 1.00 & 1.00 \\
\hline $\mathrm{Mp}$ & 1.07 & 1.20 & 0.00 & 5.00 & 59 & 1.00 & 0.00 \\
\hline Intellectualization & 1.39 & 1.61 & 0.00 & 7.00 & 104 & 1.00 & 0.00 \\
\hline $\mathrm{Zf}$ & 8.76 & 2.91 & 2.00 & 17.00 & 104 & 8.50 & 7.00 \\
\hline $\mathrm{Zd}$ & 0.72 & 3.49 & $-6 .-50$ & 13.00 & 104 & 1.00 & --- \\
\hline Blends & 2.57 & 2.06 & 0.00 & 10.00 & 88 & 2.00 & 1.00 \\
\hline Blends/R & 0.16 & 0.13 & 0.00 & 0.59 & 88 & 0.14 & 0.00 \\
\hline Col. Shading Blends & 0.31 & 0.61 & 0.00 & 3.00 & 25 & 0.00 & 0.00 \\
\hline Afr & 0.57 & 0.26 & 0.20 & 1.29 & 104 & 0.50 & 0.50 \\
\hline Popular & 3.69 & 1.59 & 0.00 & 8.00 & 101 & 4.00 & 4.00 \\
\hline $\mathrm{XA} \%$ & 0.74 & 0.14 & 0.44 & 1.00 & 104 & 0.77 & 0.93 \\
\hline WDA\% & 0.79 & 0.15 & 0.40 & 1.00 & 104 & 0.80 & 1.00 \\
\hline $\mathrm{X}+\%$ & 0.46 & 0.13 & 0.19 & 0.71 & 104 & 0.50 & --- \\
\hline $\mathrm{X}-\%$ & 0.25 & 0.13 & 0.00 & 0.56 & 103 & 0.22 & 0.07 \\
\hline $\mathrm{Xu} \%$ & 0.29 & 0.11 & 0.07 & 0.60 & 104 & 0.28 & 0.21 \\
\hline Isolate/R & 0.25 & 0.20 & 0.00 & 0.86 & 93 & 0.21 & 0.00 \\
\hline $\mathrm{H}$ & 1.70 & 1.29 & 0.00 & 5.00 & 86 & 1.00 & 1.00 \\
\hline$(\mathrm{H})$ & 0.78 & 0.84 & 0.00 & 4.00 & 58 & 1.00 & 0.00 \\
\hline $\mathrm{Hd}$ & 0.85 & 1.14 & 0.00 & 5.00 & 51 & 0.00 & 0.00 \\
\hline (Hd) & 0.36 & 0.62 & 0.00 & 2.00 & 29 & 0.00 & 0.00 \\
\hline $\mathrm{Hx}$ & 0.30 & 0.68 & 0.00 & 3.00 & 22 & 0.00 & 0.00 \\
\hline All H Contents & 3.98 & 2.27 & 0.00 & 10.00 & 100 & 4.00 & 5.00 \\
\hline A & 6.90 & 2.61 & 3.00 & 15.00 & 104 & 6.00 & 6.00 \\
\hline (A) & 0.32 & 0.52 & 0.00 & 2.00 & 30 & 0.00 & 0.00 \\
\hline Ad & 1.09 & 1.09 & 0.00 & 4.00 & 65 & 1.00 & 0.00 \\
\hline (Ad) & 0.06 & 0.23 & 0.00 & 1.00 & 6 & 0.00 & 0.00 \\
\hline An & 0.77 & 1.06 & 0.00 & 5.00 & 49 & 0.00 & 0.00 \\
\hline Art & 0.84 & 1.08 & 0.00 & 5.00 & 50 & 0.00 & 0.00 \\
\hline Ay & 0.19 & 0.46 & 0.00 & 2.00 & 17 & 0.00 & 0.00 \\
\hline $\mathrm{Bl}$ & 0.20 & 0.73 & 0.00 & 6.00 & 13 & 0.00 & 0.00 \\
\hline $\mathrm{Bt}$ & 1.27 & 1.27 & 0.00 & 6.00 & 68 & 1.00 & 0.00 \\
\hline $\mathrm{Cg}$ & 0.77 & 0.94 & 0.00 & 4.00 & 53 & 1.00 & 0.00 \\
\hline $\mathrm{Cl}$ & 0.27 & 0.68 & 0.00 & 4.00 & 18 & 0.00 & 0.00 \\
\hline Ex & 0.12 & 0.35 & 0.00 & 2.00 & 11 & 0.00 & 0.00 \\
\hline $\mathrm{Fi}$ & 0.27 & 0.67 & 0.00 & 4.00 & 19 & 0.00 & 0.00 \\
\hline $\mathrm{Fd}$ & 0.18 & 0.45 & 0.00 & 2.00 & 16 & 0.00 & 0.00 \\
\hline $\mathrm{Ge}$ & 0.10 & 0.29 & 0.00 & 1.00 & 10 & 0.00 & 0.00 \\
\hline $\mathrm{Hh}$ & 0.35 & 0.60 & 0.00 & 2.00 & 29 & 0.00 & 0.00 \\
\hline Ls & 0.69 & 0.82 & 0.00 & 3.00 & 52 & 0.50 & 0.00 \\
\hline $\mathrm{Na}$ & 0.69 & 0.94 & 0.00 & 4.00 & 45 & 0.00 & 0.00 \\
\hline $\mathrm{Sc}$ & 0.73 & 0.96 & 0.00 & 4.00 & 51 & 0.00 & 0.00 \\
\hline Sx & 0.19 & 0.62 & 0.00 & 4.00 & 12 & 0.00 & 0.00 \\
\hline $\mathrm{Xy}$ & 0.21 & 0.55 & 0.00 & 3.00 & 16 & 0.00 & 0.00 \\
\hline Id & 0.68 & 1.01 & 0.00 & 5.00 & 44 & 0.00 & 0.00 \\
\hline DV & 0.89 & 1.06 & 0.00 & 5.00 & 55 & 1.00 & 0.00 \\
\hline INCOM & 0.56 & 0.79 & 0.00 & 3.00 & 42 & 0.00 & 0.00 \\
\hline DR & 0.76 & 1.30 & 0.00 & 7.00 & 42 & 0.00 & 0.00 \\
\hline FABCOM & 0.32 & 0.59 & 0.00 & 3.00 & 28 & 0.00 & 0.00 \\
\hline DV2 & 0.05 & 0.25 & 0.00 & 2.00 & 4 & 0.00 & 0.00 \\
\hline
\end{tabular}


Tabela 12 - RIAP Estatística descritiva para 104 protocolos selecionados: R <19

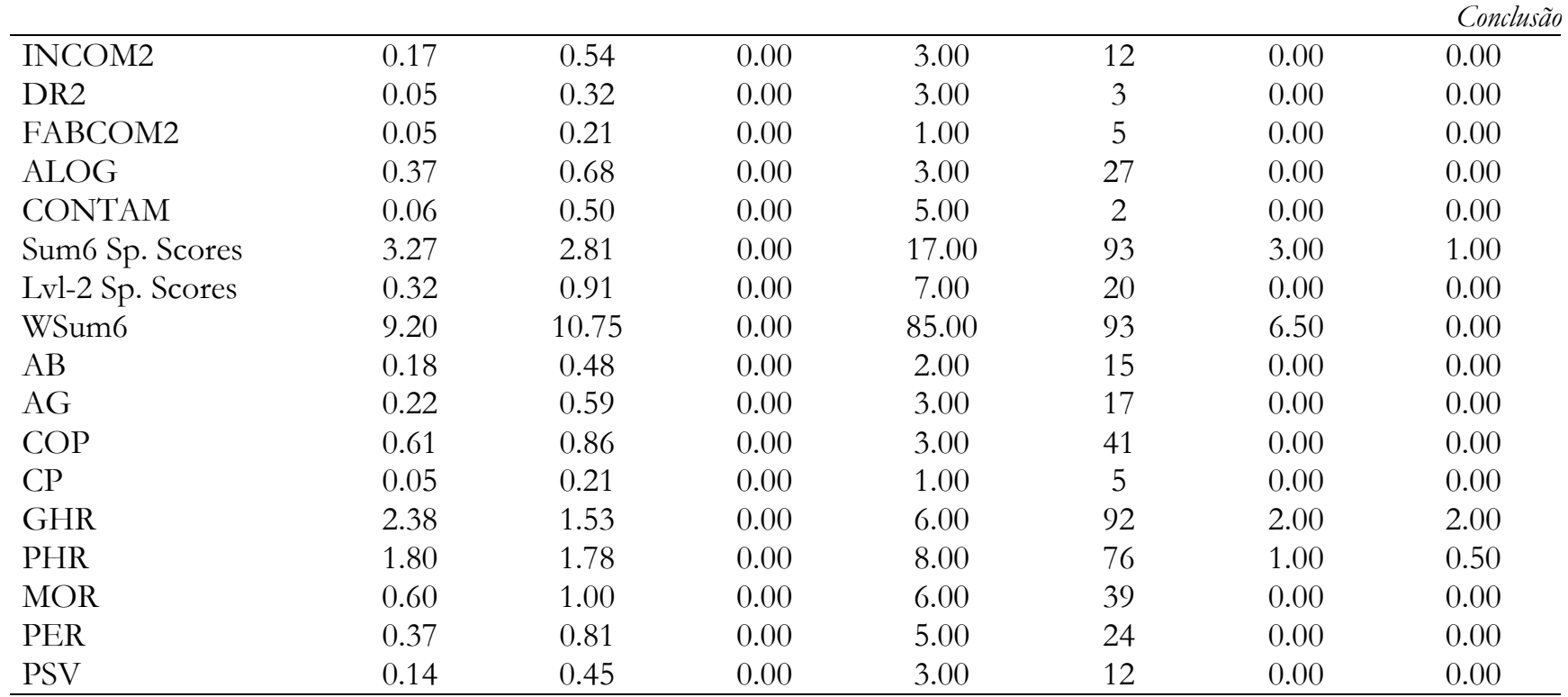

Anexo 5

Tabela 13 - RIAP Estatística descritiva para 96 protocolos selecionados: R $>18$

\begin{tabular}{|c|c|c|c|c|c|c|c|}
\hline Variável & Média & $\mathrm{DP}$ & Mínimo & Máximo & Freqüência & Mediana & Moda \\
\hline Idade & 31.46 & 9.52 & 17.00 & 58.00 & 96 & 30.00 & 25.00 \\
\hline Yrs. Ed. & 10.73 & 6.30 & 0.00 & 23.00 & 79 & 12.00 & 0.00 \\
\hline $\mathrm{R}$ & 25.45 & 6.79 & 19.00 & 50.00 & 96 & 23.00 & 22.00 \\
\hline W & 8.82 & 4.58 & 0.00 & 19.00 & 95 & 8.00 & 4.00 \\
\hline $\mathrm{D}$ & 11.41 & 4.57 & 4.00 & 27.00 & 96 & 11.00 & 9.00 \\
\hline Dd & 5.22 & 3.88 & 0.00 & 19.00 & 91 & 4.00 & 4.00 \\
\hline S & 2.15 & 2.08 & 0.00 & 10.00 & 76 & 1.50 & 1.00 \\
\hline $\mathrm{DQ}+$ & 5.42 & 3.48 & 0.00 & 15.00 & 90 & 5.00 & 4.00 \\
\hline DQo & 17.65 & 6.64 & 5.00 & 41.00 & 96 & 17.00 & 17.00 \\
\hline $\mathrm{DQv}$ & 2.02 & 2.15 & 0.00 & 12.00 & 72 & 1.00 & 1.00 \\
\hline $\mathrm{DQv} /+$ & 0.36 & 0.88 & 0.00 & 6.00 & 22 & 0.00 & 0.00 \\
\hline $\mathrm{FQx}+$ & 0.20 & 0.49 & 0.00 & 2.00 & 15 & 0.00 & 0.00 \\
\hline FQxo & 10.49 & 3.69 & 3.00 & 23.00 & 96 & 10.00 & --- \\
\hline FQxu & 7.93 & 3.92 & 1.00 & 22.00 & 96 & 7.00 & 7.00 \\
\hline FQx- & 6.35 & 2.78 & 1.00 & 16.00 & 96 & 6.00 & 6.00 \\
\hline FQx_none & 0.48 & 1.01 & 0.00 & 6.00 & 29 & 0.00 & 0.00 \\
\hline MQual + & 0.09 & 0.29 & 0.00 & 1.00 & 9 & 0.00 & 0.00 \\
\hline MQual o & 1.86 & 1.58 & 0.00 & 8.00 & 79 & 2.00 & 1.00 \\
\hline MQual u & 0.74 & 0.95 & 0.00 & 5.00 & 49 & 1.00 & 0.00 \\
\hline MQual - & 0.81 & 1.25 & 0.00 & 5.00 & 39 & 0.00 & 0.00 \\
\hline MQual none & 0.04 & 0.41 & 0.00 & 4.00 & 1 & 0.00 & 0.00 \\
\hline S- & 0.69 & 0.96 & 0.00 & 4.00 & 42 & 0.00 & 0.00 \\
\hline M & 3.55 & 3.05 & 0.00 & 16.00 & 88 & 3.00 & 2.00 \\
\hline FM & 3.81 & 2.63 & 0.00 & 13.00 & 89 & 3.00 & 2.50 \\
\hline $\mathrm{m}$ & 1.63 & 1.53 & 0.00 & 7.00 & 70 & 1.00 & 1.00 \\
\hline $\mathrm{FM}+\mathrm{m}$ & 5.44 & 3.34 & 0.00 & 15.00 & 94 & 5.00 & 4.00 \\
\hline
\end{tabular}


Tabela 13 - RIAP Estatística descritiva para 96 protocolos selecionados: R>18

\begin{tabular}{|c|c|c|c|c|c|c|c|}
\hline $\mathrm{FC}$ & 1.81 & 1.76 & 0.00 & 8.00 & 72 & 1.00 & 1.00 \\
\hline $\mathrm{CF}$ & 1.07 & 1.19 & 0.00 & 5.00 & 58 & 1.00 & 0.00 \\
\hline $\mathrm{C}$ & 0.48 & 0.88 & 0.00 & 4.00 & 29 & 0.00 & 0.00 \\
\hline $\mathrm{Cn}$ & 0.00 & 0.00 & 0.00 & 0.00 & 0 & 0.00 & 0.00 \\
\hline $\mathrm{FC}+\mathrm{CF}+\mathrm{C}+\mathrm{Cn}$ & 3.36 & 2.35 & 0.00 & 10.00 & 86 & 3.00 & --- \\
\hline WSum C & 2.70 & 1.96 & 0.00 & 7.50 & 86 & 2.50 & --- \\
\hline Sum C' & 1.44 & 1.34 & 0.00 & 6.00 & 72 & 1.00 & 1.00 \\
\hline Sum T & 0.46 & 0.87 & 0.00 & 4.00 & 28 & 0.00 & 0.00 \\
\hline Sum V & 0.71 & 1.03 & 0.00 & 5.00 & 41 & 0.00 & 0.00 \\
\hline Sum Y & 2.41 & 2.33 & 0.00 & 13.00 & 75 & 2.00 & 2.00 \\
\hline Sum Shading & 5.01 & 3.91 & 0.00 & 19.00 & 93 & 4.00 & 3.00 \\
\hline $\mathrm{Fr}+\mathrm{rF}$ & 0.56 & 1.06 & 0.00 & 5.00 & 27 & 0.00 & 0.00 \\
\hline FD & 0.60 & 1.04 & 0.00 & 5.00 & 33 & 0.00 & 0.00 \\
\hline $\mathrm{F}$ & 11.14 & 5.16 & 3.00 & 26.00 & 96 & 11.00 & 7.00 \\
\hline Pair & 6.54 & 3.55 & 1.00 & 18.00 & 96 & 6.00 & 5.00 \\
\hline $3 r+(2) / \mathrm{R}$ & 0.33 & 0.16 & 0.04 & 0.86 & 96 & 0.33 & 0.14 \\
\hline Lambda & 1.07 & 1.00 & 0.17 & 5.67 & 96 & 0.74 & 0.67 \\
\hline EA & 6.25 & 4.03 & 0.00 & 23.50 & 95 & 5.25 & 5.00 \\
\hline es & 10.45 & 5.61 & 1.00 & 28.00 & 96 & 9.00 & 6.00 \\
\hline D Score & -1.29 & 1.82 & -7.00 & 4.00 & 96 & -1.00 & 0.00 \\
\hline Adj D Score & -0.50 & 1.46 & -6.00 & 6.00 & 96 & 0.00 & 0.00 \\
\hline a (active) & 4.71 & 3.36 & 0.00 & 18.00 & 92 & 4.00 & 3.00 \\
\hline $\mathrm{p}$ (passive) & 4.34 & 3.08 & 0.00 & 16.00 & 90 & 4.00 & 3.00 \\
\hline $\mathrm{Ma}$ & 1.69 & 1.92 & 0.00 & 12.00 & 69 & 1.00 & 1.00 \\
\hline $\mathrm{Mp}$ & 1.91 & 2.06 & 0.00 & 13.00 & 70 & 1.00 & 0.00 \\
\hline Intellectualization & 2.57 & 3.67 & 0.00 & 26.00 & 96 & 2.00 & 0.00 \\
\hline Zf & 11.66 & 4.75 & 1.00 & 22.00 & 96 & 11.00 & 10.00 \\
\hline $\mathrm{Zd}$ & -1.81 & 5.57 & -19.00 & 9.00 & 96 & -1.50 & --- \\
\hline Blends & 3.68 & 3.20 & 0.00 & 16.00 & 83 & 3.00 & 1.00 \\
\hline Blends/R & 0.15 & 0.12 & 0.00 & 0.62 & 83 & 0.12 & 0.00 \\
\hline Col. Shading Blends & 0.43 & 0.67 & 0.00 & 3.00 & 32 & 0.00 & 0.00 \\
\hline Afr & 0.55 & 0.25 & 0.20 & 1.75 & 96 & 0.50 & --- \\
\hline Popular & 4.48 & 1.88 & 1.00 & 9.00 & 96 & 4.00 & 4.50 \\
\hline $\mathrm{XA} \%$ & 0.73 & 0.12 & 0.29 & 0.95 & 96 & 0.73 & --- \\
\hline WDA\% & 0.77 & 0.12 & 0.38 & 1.00 & 96 & 0.78 & 0.75 \\
\hline $\mathrm{X}+\%$ & 0.42 & 0.11 & 0.12 & 0.67 & 96 & 0.43 & --- \\
\hline $\mathrm{X}-\%$ & 0.25 & 0.11 & 0.04 & 0.62 & 96 & 0.25 & 0.27 \\
\hline $\mathrm{Xu} \%$ & 0.30 & 0.10 & 0.05 & 0.53 & 96 & 0.32 & 0.32 \\
\hline Isolate/R & 0.22 & 0.13 & 0.00 & 0.65 & 93 & 0.22 & 0.26 \\
\hline $\mathrm{H}$ & 2.39 & 1.95 & 0.00 & 9.00 & 84 & 2.00 & 1.00 \\
\hline$(\mathrm{H})$ & 1.10 & 1.28 & 0.00 & 7.00 & 57 & 1.00 & 0.00 \\
\hline $\mathrm{Hd}$ & 1.96 & 2.06 & 0.00 & 9.00 & 72 & 1.00 & 1.00 \\
\hline (Hd) & 0.66 & 1.07 & 0.00 & 5.00 & 34 & 0.00 & 0.00 \\
\hline $\mathrm{Hx}$ & 0.80 & 2.02 & 0.00 & 17.00 & 30 & 0.00 & 0.00 \\
\hline All H Contents & 6.91 & 4.60 & 0.00 & 26.00 & 95 & 6.00 & 3.00 \\
\hline A & 8.69 & 2.93 & 3.00 & 17.00 & 96 & 9.00 & 10.00 \\
\hline (A) & 0.31 & 0.58 & 0.00 & 2.00 & 24 & 0.00 & 0.00 \\
\hline Ad & 2.18 & 1.80 & 0.00 & 9.00 & 81 & 2.00 & 1.00 \\
\hline (Ad) & 0.10 & 0.31 & 0.00 & 1.00 & 10 & 0.00 & 0.00 \\
\hline An & 1.74 & 1.98 & 0.00 & 10.00 & 65 & 1.00 & 0.00 \\
\hline Art & 1.11 & 1.33 & 0.00 & 6.00 & 53 & 1.00 & 0.00 \\
\hline
\end{tabular}


Tabela 13 - RIAP Estatística descritiva para 96 protocolos selecionados: R>18

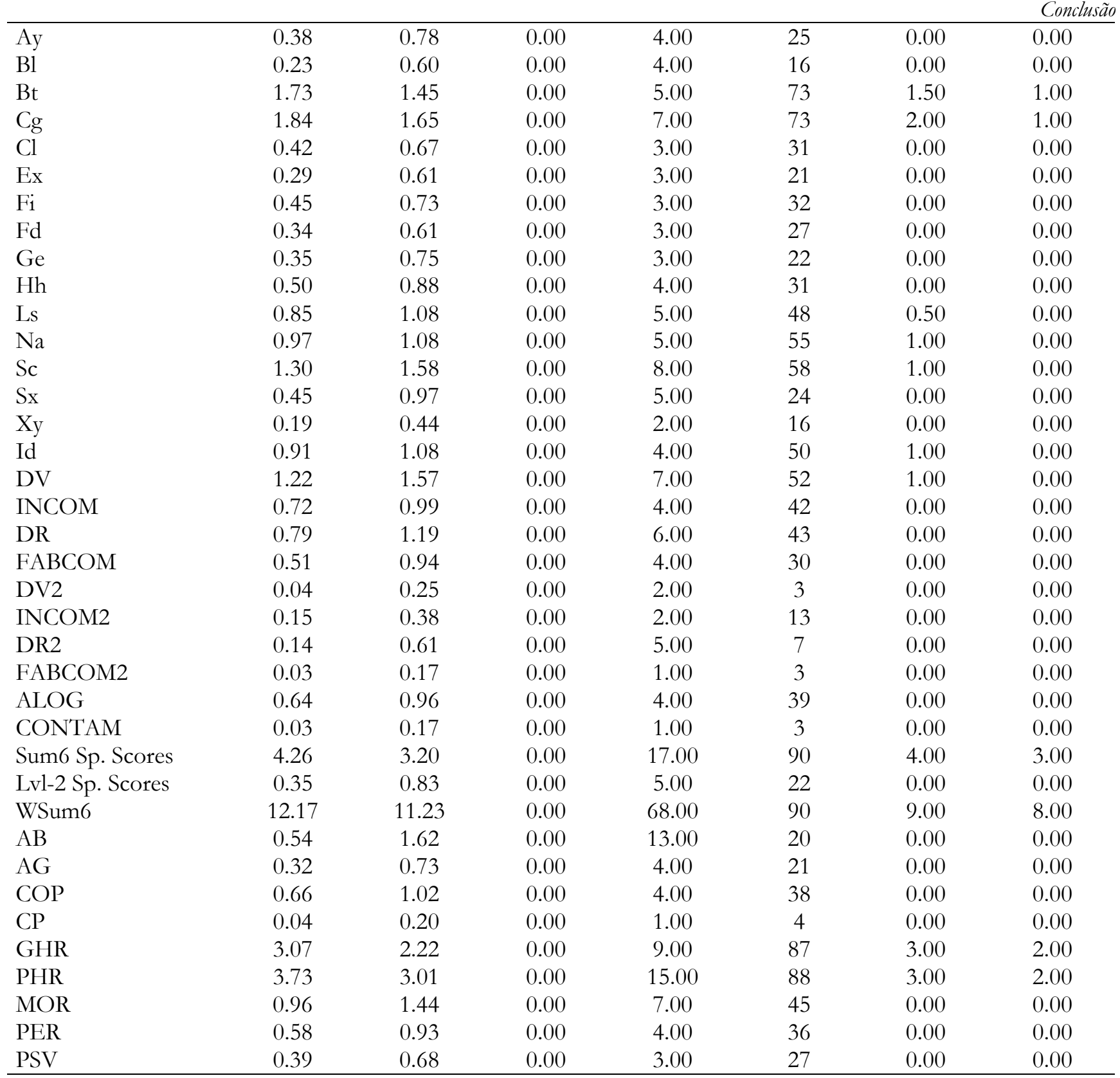

Sobre a autora:

Regina Sonia Gattas Fernandes do Nascimento é psicóloga, mestre em Psicologia Social e doutora em Psicologia Clínica, professora associada da Faculdade de Psicologia da PUC-SP e da SBRo em São Paulo, coordenadora do LEPSI (Laboratório de Estudos em Psicodiagnóstico) da PUC-SP e secretária da SBRo. 
